\section{REVISTA BRASILEIRA DE QUALIDADE DE VIDA}

\title{
Versão francesa da ferramenta para o cálculo dos escores e estatística descritiva do WHOQOL-bref através do Microsoft Excel
}

\section{French version of the tool for calculation of scores and descriptive statistics of WHOQOL-bref through Microsoft Excel}

\author{
Version française de l'outil de calcul Microsoft Excel des scores et des \\ statistiques descriptives de WHOQOL-bref
}

\author{
Bruno Pedroso \\ Universidade Estadual de Ponta Grossa - UEPG - Ponta Grossa - Brasil \\ brunops3@brturbo.com.br
}

A subjetividade da qualidade de vida possibilita a formulação de conceitos distintos para esta variável. Atrelada a tal perspectiva, a multidimensionalidade da qualidade de vida proporciona que se contemplem dimensões, as quais, sob o amparo de diferentes pesquisadores, podem ser distintas.

O fato de não haver um consenso sobre o conceito de qualidade de vida constitui um grande problema no desenvolvimento de instrumentos de avaliação da qualidade de vida, ao passo que não é possível afirmar, com clareza, quais elementos estes instrumentos estão mensurando (FLECK, 2008).

Partindo dessa premissa, o ponto inicial para a construção do instrumento de avaliação da qualidade de vida da Organização Mundial da Saúde (OMS), foi conceituar qualidade de vida. No conceito adotado, qualidade de vida é entendida como a "[...] percepção do indivíduo de sua posição na vida no contexto da cultura e sistema de valores nos quais ele vive e em relação aos seus objetivos, expectativas, padrões e preocupações" (FLECK, 2008 p. 25).

Frente a esse conceito, a OMS empenhou-se na construção dos instrumentos WHOQOL, que avaliam a qualidade de vida de forma global (WHOQOL-100, WHOQOL-bref e WHOQOL-8) como também em função de fatores e/ou grupos específicos (WHOQOL-HIV, WHOQOL-HIVbref, WHOQOL-OLD, WHOQOL-SRPB, WHOQOL-DIS, WHOQOL-Children e WHOQOLPain).

A OMS sugere a utilização do software estatístico SPSS para o cálculo dos resultados dos instrumentos WHOQOL, sendo requerida a inserção da sintaxe para o cálculo dos escores dos domínios e facetas de cada instrumento.

O rigor metodológico utilizado no processo de construção e validação dos instrumentos WHOQOL possibilita a obtenção de características psicométricas satisfatórias, perfazendo com que os resultados das aplicações de tais instrumentos sejam válidos, consistentes e fidedignos às populações estudadas. 
Entretanto, ainda que os instrumentos WHOQOL sejam os instrumentos de avaliação da qualidade de vida mais utilizados no mundo e apresentem uma difusão ímpar, o emprego do software SPSS para o cálculo dos resultados, constitui um fator limitante para a sua utilização.

Para tanto, empenhou-se na construção de uma ferramenta construída a partir do software Microsoft Excel para o cálculo dos escores e estatística descritiva do instrumento WHOQOL-100, a qual foi publicada em Pedroso, Pilatti e Reis (2009).

Em defronte a uma série de solicitações para a construção da mesma ferramenta com direcionamento ao WHOQOL-bref, posteriormente esta fora construída e publicada em Pedroso et al. (2010a). Em menor escala, houve solicitações para a construção de uma ferramenta direcionada ao WHOQOL-OLD, a qual foi publicada em Pedroso, Pilatti e Gutierrez (2010).

Tendo sido constatado que a aceitação das referidas ferramentas havia sido consolidada, não se aguardou mais solicitações para a construção das mesmas ferramentas para os instrumentos WHOQOL-SRPB e WHOQOL-HIV/WHOQOL-HIV-bref, as quais foram publicadas em Pedroso et al. (2012a) e Pedroso et al. (2012b), respectivamente.

Não obstante às solicitações de pesquisadores brasileiros, houve solicitações da mesma natureza oriundas de autores estrangeiros, questionando se havia uma versão das referidas ferramentas no idioma inglês. Estas foram, portanto, traduzidas para o idioma inglês e disponibilizadas em Pedroso et al. (2010b).

Recentemente, fora solicitado pelo pesquisador Frédéric Widart, da Université de Liège, na Bélgica, a confecção da versão francesa da ferramenta para o cálculo dos escores e estatística descritiva do WHOQOL-bref. Com a sua ajuda, esta vem a ser disponibilizada por meio da presente publicação, podendo ser obtida por meio do sítio eletrônico http://www.brunopedroso.com.br/whoqol-fr.html.

O desenvolvimento das ferramentas com direcionamento aos instrumentos WHOQOL-8, WHOQOL-DIS, WHOQOL-Children e WHOQOL-Pain segue em curso e em breve estas também estarão disponibilizadas na literatura.

\section{Referências}

FLECK, M. P. A. Problemas conceituais em qualidade de vida. In: FLECK, M. P. A. (Org.). A avaliação de qualidade de vida: guia para profissionais da saúde. Porto Alegre: Artmed, 2008.

PEDROSO, B.; PILATTI, L. A.; GUTIERREZ, G. L. Cálculo dos escores e estatística descritiva do WHOQOL-OLD através do Microsoft Excel. Geriatria \& Gerontologia, Rio de Janeiro, v. 4, n. 4, p. 214-219, out./dez. 2010.

PEDROSO, B.; PILATTI, L. A.; REIS, D. R. Cálculo dos escores e estatística descritiva do WHOQOL-100 utilizando o Microsoft Excel. Revista Brasileira de Qualidade de Vida, Ponta Grossa, v. 1, n. 1, p. 23-32, jan./jul. 2009. Crossef

PEDROSO, B. et al. Cálculo dos escores e estatística descritiva do WHOQOL-bref através do Microsoft Excel. Revista Brasileira de Qualidade de Vida, Ponta Grossa, v. 2, n. 1, p. 31-36, jan./jun. 2010a. crossef

PEDROSO, B. et al. Calculation of scores and descriptive statistics for WHOQOL instruments using Microsoft Excel. Actividad Física y Ciencias, Maracay, v. 2, n. 2, p. 01-23, jul./dez. 2010 b.

PEDROSO, B. et al. Qualidade de vida, espiritualidade, religião e crenças pessoais: análise do instrumento WHOQOL-SRPB. Revista Eletrônica Fafit/Facic, Itararé, v. 3, n. 1, p. 01-12, jan./jun. 2012a. 
PEDROSO, B. et al. Avaliação da qualidade de vida de portadores de HIV/AIDS: uma visão geral dos instrumentos WHOQOL-HIV e WHOQOL-HIV-bref. Conexões, Campinas, v. 10, n. 1, p. 5069 , jan./abr. 2012b. 